Revue d'histoire de l'Amérique française

\title{
Diversité institutionnelle et fréquentation scolaire dans l'île de Montréal en 1825 et en 1835
}

\section{Andrée Dufour}

Volume 41, numéro 4, printemps 1988

URI : https://id.erudit.org/iderudit/304614ar

DOI : https://doi.org/10.7202/304614ar

Aller au sommaire du numéro

Éditeur(s)

Institut d'histoire de l'Amérique française

ISSN

0035-2357 (imprimé)

1492-1383 (numérique)

Découvrir la revue

Citer cet article

Dufour, A. (1988). Diversité institutionnelle et fréquentation scolaire dans l'île de Montréal en 1825 et en 1835. Revue d'histoire de l'Amérique française, 41(4), 507-535. https://doi.org/10.7202/304614ar
Résumé de l'article

Cet article examine un aspect jusqu'ici peu étudié de l'histoire de l'éducation au Québec dans le premier tiers du $19^{\mathrm{e}}$ siècle : la fréquentation scolaire. Il s'appuie sur deux manuscrits inédits de Jacques Viger dans lesquels sont recensés tous les établissements d'enseignement que comptait l'île de Montréal en 1825 et en 1835.

L'île de Montréal constitue donc le terrain de cette étude qui comprend trois volets principaux. Dans un premier temps, l'étude fait état de la densité et surtout de la diversité du réseau scolaire montréalais d'alors. Puis elle considère la fréquentation scolaire des citadins et des ruraux, des filles et des garçons, des Canadiens français et des anglophones en fonction du ratio école-habitants et du nombre d'écoles mises à la disposition de ces clientèles par les différents éducateurs. En dernier lieu, elle tente de voir l'incidence du coût des études et de la gratuité scolaire sur la fréquentation des écoles.

L'article veut ainsi reconsidérer l'" apathie ", l'« indifférence " de la population à l'égard de l'instruction. Il veut également montrer que la fréquentation scolaire, prise ici comme un indice de l'accessibilité à l'instruction, fut, dans un contexte de vive rivalité, davantage régie par l'offre institutionnelle et par des facteurs économiques.
Tous droits réservés @ Institut d'histoire de l'Amérique française, 1988

Ce document est protégé par la loi sur le droit d'auteur. L'utilisation des services d'Érudit (y compris la reproduction) est assujettie à sa politique d'utilisation que vous pouvez consulter en ligne.

https://apropos.erudit.org/fr/usagers/politique-dutilisation/ 


\title{
DIVERSITÉ INSTITUTIONNELLE ET FRÉQUENTATION SCOLAIRE DANS L'ÎLE DE MONTRÉAL EN 1825 ET EN $1835^{1}$
}

\author{
ANDRÉE DUFOUR \\ Département d'histoire \\ Université du Québec à Montréal
}

\section{RÉSUMÉ}

Cet article examine un aspect jusqu'ici peu étudié de l'histoire de l'éducation au Québec dans le premier tiers du 19e siècle: la fréquentation scolaire. Il s'appuie sur deux manuscrits inédits de Jacques Viger dans lesquels sont recensés tous les établissements d'enseignement que comptait l'île de Montréal en 1825 et en 1835.

L'île de Montréal constitue donc le terrain de cette étude qui comprend trois volets principaux. Dans un premier temps, l'étude fait état de la densité et surtout de la diversité du réseau scolaire montréalais d'alors. Puis elle considère la fréquentation scolaire des citadins et des ruraux, des filles et des garçons, des Canadiens français et des anglophones en fonction du ratio écolehabitants et du nombre d'écoles mises à la disposition de ces clientèles par les différents éducateurs. En dernier lieu, elle tente de voir l'incidence du coût des études et de la gratuité scolaire sur la fréquentation des écoles.

L'article veut ainsi reconsidérer l'«apathie», l'«indifférence» de la population à l'égard de l'instruction. Il veut également montrer que la fréquentation scolaire, prise ici comme un indice de l'accessibilité à l'instruction, fut, dans un contexte de vive rivalité, davantage régie par l'offre institutionnelle et par des facteurs économiques.

\section{ABSTRACT}

This article examines an aspect of the history of education in Quebec in the first third of the nineteenth century which has heretofore received little attention from historians: school attendance. It is based on two unpublished manuscripts by Jacques Viger which list all of the teaching establishments of the island of Montreal in 1825 and in 1835 respectively.

The island of Montreal is thus the setting for this three-part-study. The study begins by showing that the components of the island's school system were both numerous and particularly diverse. It then goes on to discuss and compare school attendance of urban and rural populations, of boys and girls, of francophones and anglophones, taking into account the number of schools

1 Cet article reprend une partie de notre mémoire de maîtrise, Le réseau scolaire de l'île de Montréal, 1825-1835 (histoire) Université du Québec à Montréal, 1987. Il constitue également une revision de notre communication, «La fréquentation scolaire dans l'île de Montréal en 1825 et en 1835», présentée au Congrès de l'Institut d'histoire de l'Amérique française, Montréal, octobre 1987. Nous tenons à remercier Nadia Fahmy-Eid et Fernande Roy ainsi que les deux évaluateurs de la Revue d'histoire de l'Amérique française pour leurs judicieux commentaires concernant la rédaction de ce texte. 
per capita as well as the number of schools operated by different educators. Finally, this study attempts to determine the manner in which the cost of education influenced attendance.

This article interprets in a new way lower canadian's attitudes toward education. Instead of "apathy" and "indifference», the availability of specific types of schools and economic factors are shown to have been the prime determinants of school attendance.

La période 1801-1836, qui voit les premières tentatives d'établir un système d'enseignement public au Bas-Canada, constitue sans conteste une période marquante dans l'histoire de l'éducation au Québec. Plusieurs historiens s'y sont intéressés, notamment Lionel Groulx et Fernand Ouellet. Ainsi, le premier mettait en évidence l' "offensive» des autorités coloniales contre l'enseignement français et les audacieux et fructueux efforts de «reconstruction» de l'Église et des parlementaires bas-canadiens ${ }^{2}$. Quant au second, soucieux d'illustrer la lutte que se livrèrent l'État et l'Église pour le contrôle de l'éducation, il évoquait en particulier les motifs qui avaient amené la bourgeoisie anglophone, les autorités gouvernementales, puis les classes moyennes canadiennesfrançaises et, enfin, le haut clergé à s'intéresser au problème de l'instruction publique ${ }^{3}$. Dans ces études où dominent les conflits entourant les lois scolaires, comme d'ailleurs dans les nombreux travaux de LouisPhilippe Audet ${ }^{4}$, peu de place est faite à l'important problème de la fréquentation scolaire, si ce n'est pour en noter la stagnation ou l'augmentation après l'adoption des lois et pour souligner l'indifférence, l'apathie des parents vis-à-vis de l'instruction de leurs enfants ${ }^{5}$. Dans un article plus récent, intitulé «The Pattern of Literacy in Quebec, 1745$1899{ }^{6}$ et particulièrement axé sur l'alphabétisation, Allan Greer évoquait rapidement le problème de la fréquentation scolaire pour expliquer par des raisons culturelles, religieuses essentiellement, son taux moindre, en 1836, chez les francophones et chez les filles notamment. Question centrale au milieu du 19e siècle pour les promoteurs d'un système d'instruction publique au Haut-Canada, la fréquentation scolaire a cependant fait l'objet depuis les quinze dernières années de nombreuses études au Canada anglais ${ }^{7}$. Souvent centrées sur les clientèles

2 Lionel Groulx, L'enseignement français au Canada. Dans le Québec (Depuis les débuts de la colonie jusqu'à nos jours) (Montréal, Librairie d'Action canadienne-française, 1931), chapitres 1,2 et 3.

3 Fernand Ouellet, «L'enseignement primaire: responsabilité des Églises ou de l'État (18011836)», Éléments d'histoire sociale du Bas-Canada (Montréal, Hurtubise HMH, coll. «Cahiers du Québec», 1972), 259-277.

4 Entre autres, ses ouvrages sur Le système scolaire de la Province de Québec, III: L'Institution royale. Les débuts: 1801-1825 et IV: L'Institution royale. Le déclin: 1825-1836 (Québec, Presses de l'Université Laval, 1952).

5 L. Groulx, op. cit., 165, 266-268; F. Ouellet, op. cit., 263; L.-P. Audet, Histoire de l'enseignement, I: 1608-1840 (Montréal/Toronto, Holt, Rinehart et Winston, 1971), 363-365.

6 Article paru dans Histoire sociale/Social History, 11,22 (novembre 1978): 295-335.

7 Parmi celles-ci, mentionnons, entre autres, trois études reprises dans Michael B. Katz et Paul H. Mattingly, dir., Education and Social Change. Themes from Ontario's Past (New York, 
scolaires, ces études ont mis en évidence l'importance des attitudes familiales en matière de scolarisation, attitudes surtout régies par les nécessités économiques.

Le présent article a pour objectif premier d'établir les taux de fréquentation scolaire de la période 1801-1836 selon le différentiel ruralurbain, l'appartenance de sexe et la langue de la clientèle et de suggérer quelques explications aux données retrouvées. On ne peut nier que des facteurs culturels et la demande des parents ont joué un rôle important dans les taux de scolarisation dont nous ferons état selon les variables mentionnées précédemment. Dans le premier tiers du $19 \mathrm{e}$ siècle, marqué au Bas-Canada par de multiples rivalités entourant l'instruction primaire, nous croyons cependant que la fréquentation scolaire, qui témoigne de l'accessibilité à l'enseignement, était davantage une question de disponibilité des écoles, en d'autres mots d'offre institutionnelle. Elle était souvent reliée à la présence et à la proximité des écoles ainsi qu'au coût de l'enseignement. Nous examinerons donc la fréquentation scolaire en fonction du ratio école-habitants ${ }^{8}$, puis nous tenterons de voir l'incidence du coût des études et de la gratuité scolaire sur la scolarisation. Par ailleurs, cette disponibilité était largement dépendante des autorités coloniales, de l'Église, des classes moyennes canadiennes-françaises, de la bourgeoisie anglophone, bref des principaux éducateurs mis en évidence par l'historiographie. Ainsi pensonsnous pouvoir déjà montrer que l'apathie, l'indifférence des Canadiens français en matière d'éducation mériteraient d'être reconsidérées. L'île de Montréal a été notre terrain d'étude car elle constitue un terrain bien circonscrit et, à la fin de la période 1801-1836, un milieu diversifié que nous évoquerons brièvement. Notre choix a été aussi dicté par des raisons de disponibilité de sources, sources dont nous donnons ici un bref aperçu.

\section{LES SOURCES}

Notre étude s'appuie essentiellement sur deux documents manuscrits inédits, intitulés Enseignement public dans le Comté de Montréal, en 1825 et Enseignement public dans le Comté de Montréal, en $1835^{9}$. Il s'agit de deux recensements des établissements d'enseignement (maison, école, couvent, académie, pensionnat, etc.) -- d'importance variée

\footnotetext{
New York University Press, 1975); H. P. Bamman, «Patterns of School Attendance in Toronto, 1844-1878: some Spatial Considerations»; M. B. Katz, «Who Went to School»; I. E. Davey, «School Reform and School Attendance: the Hamilton Central School, 1853-1861».

8 Il y avait bien sûr école et école: les écoles différaient par la taille et aussi par le niveau. Dans la plupart, sinon dans toutes, les «grandes écoles» y compris, les élèves pouvaient toutefois recevoir un enseignement primaire.

9 Manuscrits 018 et 018a, Fonds Verreau, Archives du Séminaire de Québec. Pour une description et une critique plus détaillées de cette source, nous renvoyons pour l'instant le lecteur à notre mémoire de maîtrise.
} 
— que comptait l'île en 1825 et en $1835^{10}$. Précisons que l'expression «enseignement public» doit être comprise dans le sens d'un enseignement dispensé ailleurs que dans la famille.

Le dénombrement des écoles de 1825 a été effectué par l'inspecteur des chemins Jacques Viger lors du recensement du Bas-Canada de 1825 pour lequel lui et le notaire Louis Guy avaient été nommés commissaires recenseurs pour le comté de Montréal ${ }^{11}$; celui de 1835 fut réalisé par Viger seul lors de son passage à la mairie de Montréal. Auteur de la célèbre Saberdache ${ }^{12}$ et des Tablettes Statistiques du Comté de Montréal, en $1825^{13}$, Jacques Viger est une figure bien connue des historiens. La critique de ses manuscrits sur les écoles nous a permis d'y constater le souci d'exactitude et la précision qui caractérisent Viger, un témoin par ailleurs privilégié de son époque. Parmi les nombreuses informations que contiennent les deux manuscrits, nous retrouvons: le genre de l'établissement, le nom de la personne ou des organismes qui le tiennent ou encore le soutiennent, la langue d'enseignement, le nombre et le sexe des maîtres, le nombre total d'écoliers, de garçons et de filles, d'élèves payants et non payants ainsi que l'origine ethnique et géographique des élèves. Les recensements font également état des matières enseignées, du coût des études et de la pension. Les deux tableaux récapitulatifs résument d'ailleurs bon nombre des informations recueillies, informations qui font des deux manuscrits de Viger des sources précieuses pour l'étude de la fréquentation scolaire ainsi que du réseau scolaire montréalais du premier tiers du $19 \mathrm{e}$ siècle. Nous allons nous attarder sur ce réseau après avoir souligné quelques aspects de l'île et de la cité de Montréal dans les décennies 1820 et 1830.

\section{L'ÎLE ET LA CITÉ DE MONTRÉAL}

Située au confluent de l'Outaouais et du Saint-Laurent, l'île de Montréal est à l'époque divisée en 10 paroisses ${ }^{14}$, comme le montre la carte dessinée d'après celle établie par André Jobin en 1834. Toutes ces paroisses sont essentiellement rurales, à l'exception de l'importante et populeuse Paroisse de Montréal qui comprend une zone rurale formée de cinq divisions ${ }^{15}$ et une zone urbaine, la cité de Montréal dont les

\footnotetext{
10 Pour la liste de tous ces établissements et de leurs principales caractéristiques, nous renvoyons également le lecteur à notre mémoire de maîtrise.

11 L'île de Montréal correspondait alors au comté de recensement de Montréal.

12 Inventoriée par F. Ouellet dans $R A P Q, 36-37$ (1955-1956) :33-38.

13 Ces Tablettes ont fait l'objet de publications des chercheurs du Groupe de recherche sur la société montréalaise au 19e siècle (GRSM) dont celle de Jean-Paul Bernard, Paul-André Linteau et Jean-Claude Robert, «Les Tablettes statistiques de Viger», Rapport 1972-1973 (Montréal, GRSM, 1973).

${ }_{14}$ Voir sur la carte les noms et les limites de ces paroisses.

15 Encore appelées «La Campagne».
} 
limites furent établies en 1791. Par leur proximité de la ville, les divisions sont différentes des autres parties rurales de l' $̂$ le. Aussi, les distinguerons-nous souvent des paroisses rurales (toutes les paroisses de l'île moins la Paroisse de Montréal) dans le texte et les tableaux. La cité comprend la vieille ville ${ }^{16}$ qui en constitue le coeur, le noyau. La vieille ville est entourée en 1825 de sept faubourgs ${ }^{17}$ formés de façon désordonnée et déjà trois fois plus peuplés à cette époque que la vieille ville.

Au total, avec ses 22540 habitants recensés en 1825, Montréal dépasse légèrement Québec. Devenue la métropole économique des Canadas, elle est le centre du commerce import-export et un important centre de fabrication de biens de consommation et de biens durables. Bien que variée, sa production est encore majoritairement artisanale dans les années 1820 et $1830^{18}$. Les artisans et les apprentis sont donc nombreux dans la cité. Celle-ci compte également de nombreux journaliers et domestiques composant plus de $40 \%$ de la population active. Artisans et journaliers s'établissent en majorité dans les faubourgs, alors que la vieille ville héberge davantage les membres de la bourgeoisie ${ }^{19}$. Au cours des années 1815 à 1835 plus particulièrement, une importante immigration britannique, composée en majorité d'Irlandais catholiques, souvent pauvres, entraîne une modification de la composition ethnique et linguistique de la cité. En 1825, les Canadiens français n'y constituent plus que $54,4 \%$ de la population; en 1832, les anglophones y deviennent majoritaires ${ }^{20}$.

Montréal subit également dans les décennies 1820 et 1830 , les effets de la querelle qui oppose l'évêque Jean-Jacques Lartigue aux Sulpiciens. Ceux-ci voient dans la nomination d'un évêque à Montréal une double menace: ils craignent de devoir partager leur pouvoir et appréhendent que cet ancien Sulpicien leur fasse perdre leurs privilèges

16 «La Ville» pour Viger.

17 En 1831, une loi créant la corporation municipale de la cité de Montréal divise la cité en huit quartiers qui remplacent les anciens faubourgs et la «vieille ville» scindée, elle, en quartiers est et ouest. Les frontières internes des quartiers Saint-Laurent et Saint-Louis subissent alors des modifications importantes et la petite Pointe-à-Callière est rattachée au quartier Sainte-Anne. Cependant, dans ses compilations de 1835, Viger a employé essentiellement les mêmes toponymes qu'en 1825 - seul le nom d'une division change. Ces toponymes recouvrent, comme nous incitent fortement à le croire les antécédents de statisticien de Viger et les vérifications que nous avons faites des adresses des écoles, les mêmes réalités territoriales. En conservant ces anciennes limites et les mêmes vocables, qui survivaient peut-être dans l'usage populaire, Viger voulait sans doute mieux suivre l'évolution de l'enseignement dans l'île.

18 J.-P. Bernard, P.-A. Linteau et J.-C. Robert, «La croissance démographique et spatiale de Montréal dans le 1er quart du 19e siècle», Rapport et travaux, 1973-1975 (Montréal, GRSM, 1975): 1-14.

19 Ibid., 28; J.-P. Bernard, P.-A. Linteau et J.-C. Robert. «La structure professionnelle de Montréal en 1825», RHAF, 30,3 (décembre 1976): 406.

20 H. Cowan, British Emigration to British North America (Toronto, University of Toronto Press, 1961), 2e édition, 66-67, 74, 186 et 289 surtout; J.-C. Robert, op. cit., 182-188. 


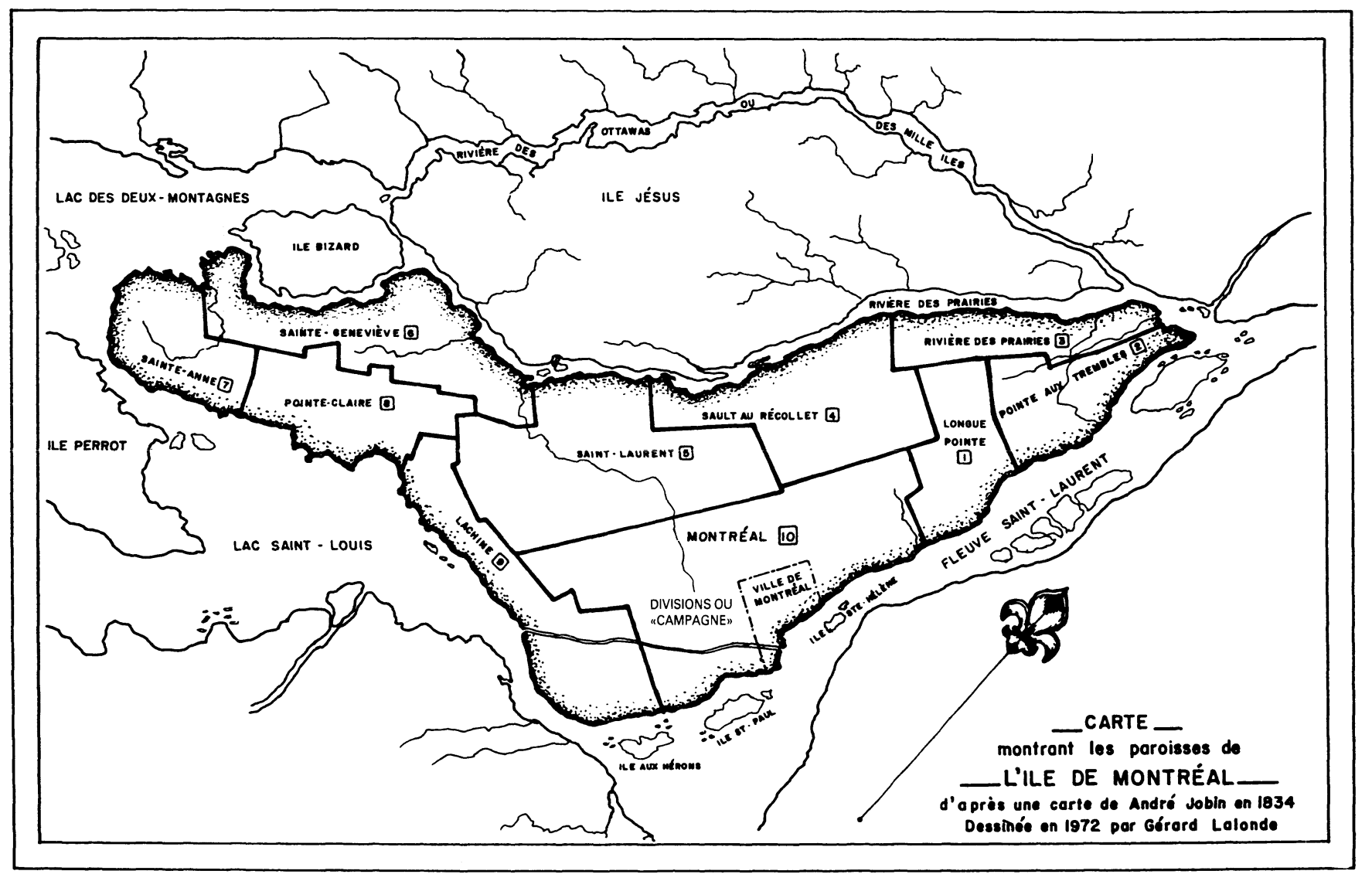

Source: Claude Perrault, Montréal en 1825, Montréal, 1977. 


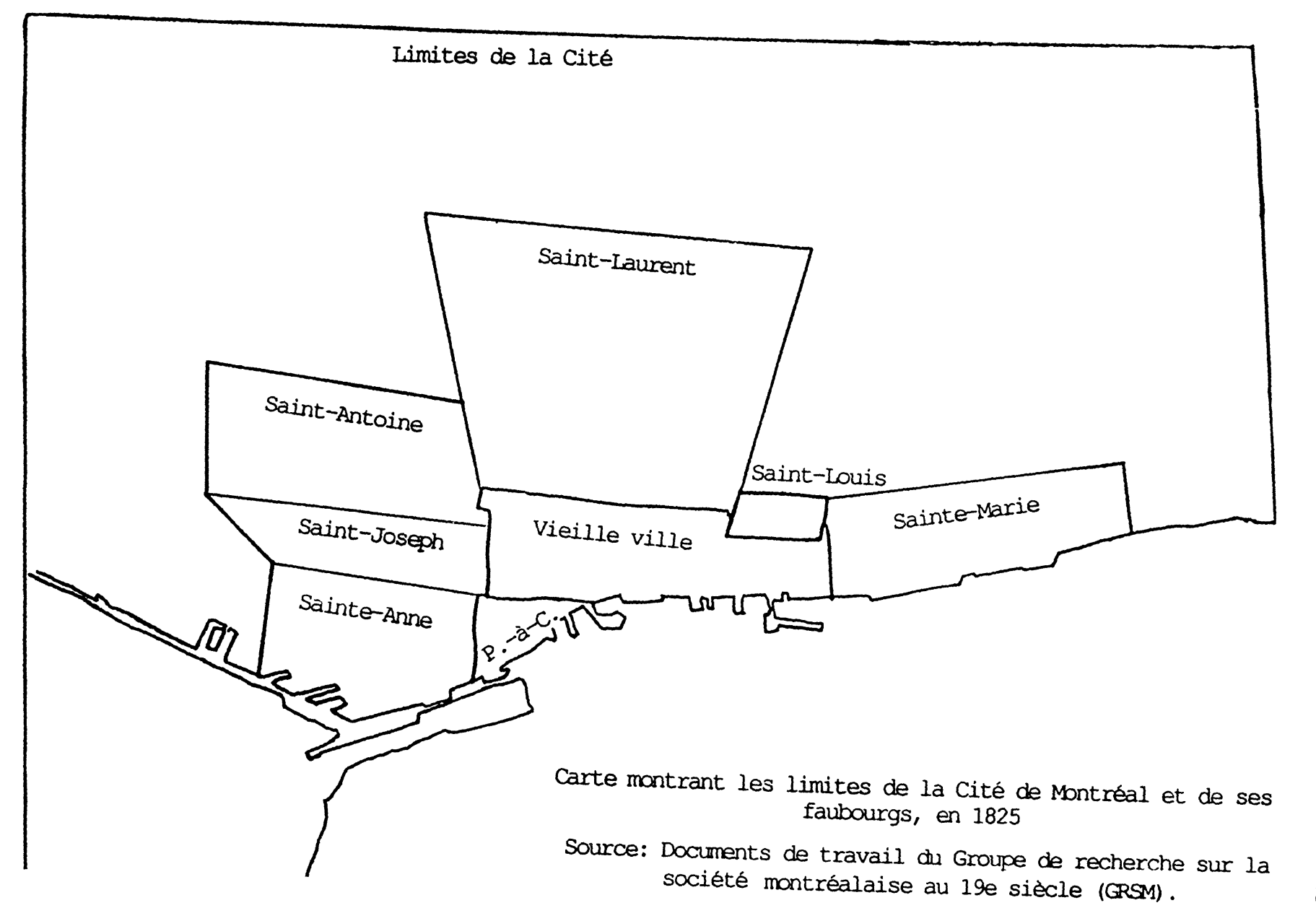


ecclésiastiques et s'empare d'une partie de leurs revenus. Expulsé du Séminaire de Montréal, J.-J. Lartigue fait ériger sa cathédrale, la cathédrale Saint-Jacques, dans le faubourg Saint-Louis. De là, malgré de nouvelles frictions avec les Sulpiciens, il parviendra à s'imposer à l'ensemble de la communauté catholique montréalaise ${ }^{21}$. Mais il sera intéressant d'observer l'incidence de cette dissension sur le développement des écoles de l'île.

\section{LE RÉSEAU SCOLAIRE MONTRÉALAIS ${ }^{22}$}

L'île de Montréal compte cinquante-quatre écoles en 1825. Sept d'entre elles se retrouvent dans les paroisses rurales, 6 dans les divisions, 24 dans les faubourgs et 17 dans la vieille ville. Pour la cité (vieille ville et faubourgs), en particulier, ces nombres apparaissent relativement élevés, du moins plus élevés que ne l'avaient révélé les sources dépouillées jusqu'ici ${ }^{23}$. En 1835 , le nombre total des écoles s'est accru, pour atteindre 89: les paroisses rurales en comptent 17, les divisions toujours 6 , les faubourgs 46 et la vieille ville 20 . Pour l'ensemble de l'île, il s'agit d'une hausse de presque $65 \%$. À première vue, l'augmentation que l'on constate en milieu rural semble bien s'inscrire dans l'accroissement général du nombre d'écoles, observé dans le BasCanada après l'adoption de la loi de 1829 créant les écoles dites «de syndics». Dans la cité, l'augmentation accompagne la croissance démographique, notamment des faubourgs, à la suite de la forte immigration britannique signalée plus haut.

D'après les informations fournies par Viger, nous avons partagé les écoles montréalaises en treize catégories différentes (voir tableaux 1 et 2). Ce partage illustre la lutte de pouvoir entourant l'instruction publique à l'époque, mais il est avant tout déterminé par la condition, la qualité des personnes ou des organismes qui dirigent ou soutiennent les écoles. Ainsi, il y a bien sûr les écoles établies en vertu des lois scolaires de 1801,1824 et 1829 , soit les écoles dites de l'Institution royale, les écoles de Fabrique et les écoles de syndics ou d'Assemblée. D'après l'origine du soutien administratif ou financier qui leur est accordé, nous distinguons également les écoles de l'évêque Lartigue, d'un curé, des Soeurs, des Sulpiciens, des Sociétés d'éducation et de bienfaisance, les écoles d'Églises autres que catholiques et, parce qu'elles sont soutenues par eux, les écoles d'habitants. Il existe égale-

21 J.-C. Robert, op. cit., 336-348.

22 Le terme «réseau» est employé, car, même s'il connote l'idée d'ensemble organisé (ce qui n'est pas encore le cas de l'enseignement au Bas-Canada de 1801 à 1836), il exprime bien la densité et la diversité qui caractérisent alors les établissements d'enseignement à Montréal.

23 L. - P. Audet, par exemple, énumère, «d'après des notes recueillies dans les Archives du Séminaire de Saint-Sulpice», 22 écoles existant à Montréal en 1828 (L.- P. Audet, Le système scolaire de la Province de Québec, V: Les écoles élémentaires dans le Bas-Canada (Québec, Presses de l'Université Laval, 1952), 46-47. 
TABLEAU 1

Répartition des écoles et des élèves selon la catégorie et le lieu des écoles, île de Montréal en 1825

\begin{tabular}{|c|c|c|c|c|c|c|c|c|c|}
\hline \multirow[b]{2}{*}{ Catégorie } & & & & & \multicolumn{2}{|c|}{ Cité } & \multirow[b]{2}{*}{$\begin{array}{r}\text { Partie } \\
\text { urbaine } \\
(F+V v)\end{array}$} & \multirow[b]{2}{*}{ Île } & \multirow[b]{2}{*}{ Île en \% } \\
\hline & & $\begin{array}{r}\text { Paroisses } \\
\text { rurales }\end{array}$ & Divisions & $\begin{array}{r}\text { Partie } \\
\text { rurale } \\
(\mathrm{Pr}+\mathrm{D})\end{array}$ & Faubourgs & $\begin{array}{r}\text { Vieille } \\
\text { ville }\end{array}$ & & & \\
\hline 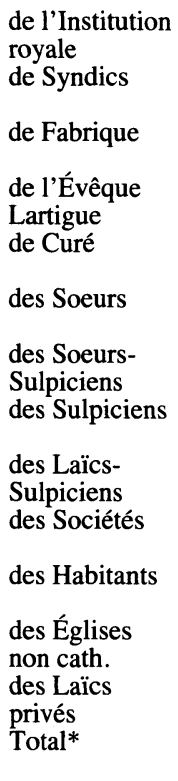 & $\begin{array}{l}\text { écoles } \\
\text { élèves } \\
\text { écoles } \\
\text { élèves } \\
\text { écoles } \\
\text { élèves } \\
\text { écoles } \\
\text { élèves } \\
\text { écoles } \\
\text { élèves } \\
\text { écoles } \\
\text { élèves } \\
\text { écoles } \\
\text { élèves } \\
\text { écoles } \\
\text { élèves } \\
\text { écoles } \\
\text { élèves } \\
\text { écoles } \\
\text { élèves } \\
\text { écoles } \\
\text { élèves } \\
\text { écoles } \\
\text { élèves } \\
\text { écoles } \\
\text { élèves } \\
\text { écoles } \\
\text { élèves }\end{array}$ & $\begin{array}{r}1 \\
28 \\
3 \\
98\end{array}$ & $\begin{array}{r}1 \\
24 \\
\\
3 \\
51 \\
6 \\
139\end{array}$ & $\begin{array}{r}1 \\
24 \\
\\
5 \\
91 \\
13 \\
353\end{array}$ & $\begin{array}{r}1 \\
60 \\
\\
1 \\
43 \\
\\
5 \\
589 \\
\\
\\
2 \\
304 \\
\\
\\
\\
\\
15 \\
418 \\
24 \\
1414\end{array}$ & $\begin{array}{r} \\
\\
\\
2 \\
300 \\
\\
\\
3 \\
191 \\
\\
\\
\\
\\
1 \\
338 \\
\\
\\
\\
\\
1 \\
70 \\
9 \\
352 \\
17 \\
1289\end{array}$ & $\begin{array}{r}1 \\
60 \\
\\
3 \\
343 \\
\\
8 \\
780 \\
\\
3 \\
642 \\
\\
\\
\\
24 \\
770 \\
41 \\
203\end{array}$ & $\begin{array}{r}1 \\
60 \\
1 \\
28 \\
6 \\
441 \\
\\
\\
10 \\
834 \\
\\
\\
\\
3 \\
642 \\
1 \\
24 \\
1 \\
70 \\
29 \\
861 \\
54 \\
3056\end{array}$ & $\begin{array}{r}1,9 \% \\
2,0 \% \\
1,9 \% \\
0,9 \% \\
11,1 \% \\
14,4 \% \\
\\
18,5 \% \\
27,3 \% \\
\\
5,5 \% \\
21,0 \% \\
1,9 \% \\
0,8 \% \\
1,9 \% \\
2,3 \% \\
53,7 \% \\
28,2 \% \\
100 \% \\
100 \%\end{array}$ \\
\hline
\end{tabular}

* Le total comprend les 10 élèves d'une préceptrice, soit $0,3 \%$ des élèves

Source: J. Viger, Enseignement public dans le Comté de Montréal, en 1825, Fonds Verreau, ASQ. 
TABLEAU 2

Répartition des écoles et des élèves selon la catégorie et le lieu des écoles, Île de Montréal en 1835

\begin{tabular}{|c|c|c|c|c|c|c|c|c|c|}
\hline \multirow{2}{*}{ Catégorie } & & \multirow[b]{2}{*}{$\begin{array}{r}\text { Paroisses } \\
\text { rurales }\end{array}$} & \multirow[b]{2}{*}{ Divisions } & \multirow[b]{2}{*}{$\begin{array}{r}\text { Partie } \\
\text { rurale } \\
(\mathrm{Pr}+\mathrm{D})\end{array}$} & \multicolumn{2}{|c|}{ Cité } & \multirow[b]{2}{*}{$\begin{array}{r}\text { Partie } \\
\text { urbaine } \\
(\mathrm{F}+\mathrm{Vv})\end{array}$} & \multirow[b]{2}{*}{ Île } & \multirow[b]{2}{*}{ Île en $\%$} \\
\hline & & & & & Faubourgs & $\begin{array}{r}\text { Vieille } \\
\text { ville }\end{array}$ & & & \\
\hline $\begin{array}{l}\text { de l'Institution } \\
\text { royale } \\
\text { de Syndics } \\
\text { de Fabrique } \\
\text { de l'Évêque } \\
\text { Lartigue } \\
\text { de Curé } \\
\text { des Soeurs } \\
\text { des Soeurs- } \\
\text { Sulpiciens } \\
\text { des Sulpiciens } \\
\text { des Laïcs- } \\
\text { Sulpiciens } \\
\text { des Sociétés } \\
\text { des Habitants } \\
\text { des Églises } \\
\text { non cath. } \\
\text { des Laïcs } \\
\text { privés } \\
\text { Total }\end{array}$ & $\begin{array}{l}\text { écoles } \\
\text { élèves } \\
\text { écoles } \\
\text { élèves } \\
\text { écoles } \\
\text { élèves } \\
\text { écoles } \\
\text { élèves } \\
\text { écoles } \\
\text { élèves } \\
\text { écoles } \\
\text { élèves } \\
\text { écoles } \\
\text { élèves } \\
\text { écoles } \\
\text { élèves } \\
\text { écoles } \\
\text { élèves } \\
\text { écoles } \\
\text { élèves } \\
\text { écoles } \\
\text { élèves } \\
\text { écoles } \\
\text { élèves } \\
\text { écoles } \\
\text { élèves } \\
\text { écoles } \\
\text { élèves }\end{array}$ & $\begin{array}{r}1 \\
25 \\
7 \\
219 \\
1 \\
19 \\
\\
1 \\
21 \\
2 \\
72\end{array}$ & $\begin{array}{r}3 \\
182 \\
1 \\
36\end{array}$ & $\begin{array}{r}1 \\
25 \\
7 \\
219 \\
1 \\
19 \\
\\
1 \\
21 \\
2 \\
72 \\
\\
3 \\
182 \\
1 \\
36\end{array}$ & $\begin{array}{rr}1 \\
212 \\
\\
1 \\
41 \\
2 \\
272 \\
5 \\
414 \\
3 \\
61 \\
4 \\
369 \\
\\
\\
1 \\
55 \\
29 \\
877 \\
46 \\
2301\end{array}$ & $\begin{array}{r}2 \\
240 \\
\\
\\
1 \\
146 \\
1 \\
43 \\
3 \\
662 \\
\\
\\
1 \\
1 \\
75 \\
11 \\
424 \\
20 \\
1620\end{array}$ & 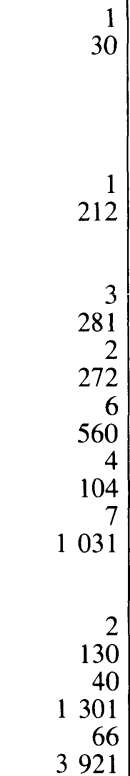 & $\begin{array}{r}2 \\
55 \\
7 \\
219 \\
1 \\
19 \\
1 \\
212 \\
1 \\
21 \\
5 \\
353 \\
2 \\
272 \\
9 \\
742 \\
5 \\
140 \\
7 \\
1031 \\
\\
\\
\\
\\
\\
\\
2 \\
130 \\
47 \\
1407 \\
89 \\
4601\end{array}$ & $\begin{array}{r}2,3 \% \\
1,2 \% \\
7,9 \% \\
4,8 \% \\
1,1 \% \\
0,4 \% \\
1,1 \% \\
4,6 \% \\
1,1 \% \\
0,4 \% \\
5,6 \% \\
7,7 \% \\
2,3 \% \\
5,9 \% \\
10,1 \% \\
16,1 \% \\
5,6 \% \\
3,1 \% \\
7,9 \% \\
22,4 \% \\
\\
2,2 \% \\
2,8 \% \\
52,8 \% \\
30,6 \% \\
100 \% \\
100 \%\end{array}$ \\
\hline
\end{tabular}

Source: J. Viger, Enseignement public dans le Comté de Montréal, en 1835, Fonds Verreau, ASQ. 
ment des établissements dits ici «laïcs-privés», car non régis par les lois scolaires, non subventionnés et dépendant uniquement de laïcs. À cet ensemble s'ajoutent deux catégories d'institutions à statut mixte ou intermédiaire. Quelques écoles regroupées dans le tableau 2 sous la mention «Soeurs-Sulpiciens» sont en effet dirigées par des religieuses tout en étant à la charge des Sulpiciens. D'autres se distinguent des écoles «des Sulpiciens» ${ }^{24}$, car bien qu'elles soient sous la «surveillance et protection du Séminaire» ${ }^{25}$, elles sont connues et recensées sous le nom de leurs directeurs laïcs. Elles pratiquent d'ailleurs des tarifs sans commune mesure avec ceux exigés par les Sulpiciens. Fruit de traditions éducatives remontant à la Nouvelle-France et de trois lois scolaires successives, le réseau scolaire de l'île de Montréal apparaît donc fort diversifié à la fin du premier tiers du 19e siècle. De plus, en 1825, quelques enfants de la campagne reçoivent, hors de l'école, l'instruction d'une préceptrice et d'un maître ambulant.

Les tableaux 1 et 2 indiquent la localisation et l'importance de ces diverses catégories d'écoles en 1825 et en 1835 . Situées dans la paroisse de Lachine et dans la vieille ville, les écoles de l'Institution royale ne sont que deux, plus de vingt ans après la loi de 1801, et ceci en comptant la Royal Grammar School de Fondation royale. L'une et l'autre accusent même un recul sensible de leur clientèle, recul compatible d'ailleurs avec le déclin de ces écoles au Bas-Canada à partir de la décennie 1830. L'État apparaît donc fort peu présent en milieu urbain au chapitre de l'enseignement.

Le succès ne semble pas non plus la caractéristique des écoles de Fabrique. Aucune n'est recensée en 1825. Créée tardivement en 1834, dans la paroisse de la Pointe-aux-Trembles, la seule qui existe en 1835 ne compte que 19 élèves. Elles ont cédé la place aux écoles de syndics qui apparaissent en milieu rural dès $1829^{26}$ et qui y regroupent près du tiers des élèves en 1835. À travers ces écoles, l'État connaît un succès certain en milieu rural.

Dans l'île, les écoles dirigées par des curés sont rares: Viger n'en a recensé qu'une, ouverte dans la paroisse de la Pointe-Claire depuis 1820. Ce qui nous porte à croire que le phénomène était peu fréquent au Bas-Canada. On compte en 1825 une petite école soutenue par huit habitants de la division Sainte-Marie. Dix ans après, on ne retrouve plus ce type d'écoles, sans doute lui aussi supplanté par les écoles de syndics.

24 En 1825, Viger emploie le terme «de paroisse» pour désigner les écoles des Sulpiciens; en 1835, pour éviter sans doute toute confusion avec les écoles de Fabrique, il utilise l'appellation «du Séminaire».

25 J. Viger, Enseignement public dans le Comté de Montréal, en 1835, Fonds Verreau 018a, Archives du Séminaire de Québec, 41.

26 Viger mentionne, en effet, que les deux écoles d'Assemblée de la paroisse du Sault-auRécollet étaient auparavant des écoles de Fabrique. 
Les religieuses, en revanche, se révèlent fort actives dans le domaine de l'enseignement, instruisant en milieu rural et en milieu urbain près de $15 \%$ des enfants scolarisés en 1825 . Elles appartiennent essentiellement à la Congrégation de Notre-Dame, les Soeurs Grises n'enseignant qu'aux orphelins et aux «enfants trouvés», recueillis dans leur hôpital de la Pointe-à-Callière. Elles ont dans la vieille ville le réputé pensionnat Notre-Dame et, à proximité, les «Petites écoles françaises», un grand externat accueillant quelque 200 enfants. Avec l'ouverture en 1833 d'écoles dans les quartiers populeux de Saint-Laurent et de Sainte-Marie, ces religieuses, soutenues par les Sulpiciens, inaugurent leur présence dans les faubourgs. Elles augmentent ainsi leur clientèle, réduite quelque peu par la fermeture du pensionnat de SaintLaurent à l'automne de 1825.

L'évêque Lartigue possède sa propre école, dite «de SaintJacques», qu'il a établie au faubourg Saint-Louis à l'automne de 1825, peu après son éviction du Séminaire de Saint-Sulpice. Formée alors d'une seule classe de 60 élèves, elle en compte quatre, dix ans plus tard, regroupant 212 écoliers et donnant à l'évêque une importance croissante dans le domaine scolaire. Cette croissance n'est sans doute pas sans inquiéter les Sulpiciens qui, depuis le $17 \mathrm{e}$ siècle, occupent à Montréal une place considérable dans l'enseignement tant par le nombre de leurs écoles que par celui des enfants qu'ils rejoignent ${ }^{27}$. Ils tiennent en outre le seul collège classique de Montréal, le Collège ou Petit Séminaire de Montréal. Les Sulpiciens ne se retrouvent que dans la Paroisse de Montréal (divisions et cité) dont ils ont la charge. Ils sont aussi absents du faubourg Saint-Louis, fief de Lartigue. Avec les écoles des religieuses et des laïcs qu'ils soutiennent et chapeautent, leur poids reste néanmoins considérable en 1835 puisqu'ils contribuent encore à l'instruction de plus du quart des écoliers de l'île.

Du côté des écoles soutenues par des Églises autres que catholiques, on ne distingue qu'un seul établissement en 1825 et deux en 1835. Ces écoles sont situées en milieu urbain et sous l'égide de l'Église presbytérienne dont les membres sont, d'après Michael Katz, les plus conscients de la nécessité d'instruire leurs enfants ${ }^{28}$. C'est peu quand on sait que les Églises anglicane et presbytérienne notamment comptent respectivement 3831 et 2982 fidèles dans la cité en 1825 et que leur nombre augmente au recensement de $1831^{29}$.

27 Voir à ce sujet l'article de Y. Poutet, «La Compagnie de Saint-Sulpice et les petites écoles de Montréal au XVIIe siècle (1657-1700)», Bulletin du Comité des Etudes (avril-juin 1961): 164-183.

${ }_{28}$ M. B. Katz, «Who Went to School» dans M. B. Katz and Paul H. Mattingly, ed., Education and Social Change. Themes from Ontario's Past (New York, New York University Press, 1975), 280.

29 J. Viger, Tablettes statistiques du Comté de Montréal, pour 1825, no 5, Dénombrement religieux, Fonds Verreau, ASQ; «Recensement et retours statistiques de la Province du BasCanada», Journaux de la Chambre d'Assemblée du Bas-Canada, 1831-1832, Appendice Oo. 
Également présentes dans les villes de Québec et de Trois-Rivières, les Sociétés d'éducation et de bienfaisance, appelées par Viger «Association de particuliers pour assister et instruire les Pauvres» et «destinées aux enfants de la classe ouvrière de toutes confessions» et aux «orphelins indigents» ${ }^{30}$, participent fortement à l'instruction des jeunes Montréalais à travers la British and Canadian School, la National School et l'Orphan Asylum, ouverts de 1819 à 1822. À elles seules, ces écoles souvent fort populeuses regroupent plus du quart des élèves de la cité. Leur nombre augmente avec l'arrivée de nombreux immigrants pauvres et l'épidémie de choléra de 1832 qui multiplie les orphelins. Ainsi, les écoles du Comité de la Montreal Recollet School Institution, de la Montreal Ladies Benevolent Society et de la Société des Dames de la Charité, la première société d'éducation francophone, ouvrent leurs portes en 1829, 1832 et 1835. La bourgeoisie montréalaise qui soutient et régit ces établissements joue donc, elle aussi, un rôle considérable dans l'éducation des jeunes citadins.

L'enseignement est cependant assuré dans une large mesure, en 1825 et davantage en 1835, par des écoles laïques privées. Déjà, L.-P. Audet avait souligné, pour la fin du $18 \mathrm{e}$ siècle et pour le début du $19 \mathrm{e}$ siècle, la présence de telles écoles, surtout anglophones, dans les villes de Québec et de Montréal ${ }^{31}$. Présentes à la campagne et surtout à la ville, elles s'avèrent cependant beaucoup plus nombreuses qu'on ne l'avait estimé jusqu'ici. Leur importance remet en question les données fournies par l'historiographie selon laquelle l'éducation dans les villes était aux mains des clercs, des Sociétés d'éducation et de quelques maîtres laïcs. Leur existence dans la cité s'explique d'ailleurs, comme celle des Sociétés d'éducation et de bienfaisance, par la présence d'une importante communauté anglophone à Montréal. Une communauté soucieuse, semble-t-il, de continuer les traditions britanniques en matière d'éducation ${ }^{32}$. Car ces écoles sont presque toutes anglophones.

En 1835, plus de la moitié des écoles laïques privées sont établies dans les faubourgs qui connaissent, avec l'immigration britannique, une croissance démographique marquée. Leur existence cependant se révèle souvent brève; la plupart apparaissent éphémères, instables. Des 29 écoles de ce type recensées en 1825, il n'en reste plus que 6 en 1835. Et 22 des 47 qui existent en 1835 ont été fondées au cours des seules années 1834 et 1835 . Elles sont en outre petites, accueillant en moyenne une trentaine d'enfants. Quelques-unes en ont même moins de dix. On comprend qu'elles soient si souvent passées inaperçues, les sources officielles ne retenant que les institutions scolaires les plus importantes

\footnotetext{
30 J. Viger, Enseignement public...1825, 12 et Enseignement public... 1835, 44.

31 L. $-\mathrm{P}$. Audet, Histoire de l' enseignement..., I: 342-343, 359.

32 L. Trenard, «L'Entre-deux siècles», Histoire mondiale de l'éducation, 2: de 1515 à 1815
} (Paris, Presses universitaires de France, 1981): 391-392. 
ou subventionnées. Il reste qu'elles reçoivent près de $30 \%$ des élèves en 1825 et 1835 et le plus grand nombre des nouveaux écoliers recencés en 1835. On peut ici émettre l'hypothèse que leur présence, qui renforce celle des Sulpiciens, des religieuses et des Sociétés d'éducation et de bienfaisance, a pu empêcher ou du moins freiner l'intervention de l'État dans la cité.

\section{FRÉQUENTATION SCOLAIRE ET ÉCOLES DISPONIBLES}

Mesurer la fréquentation scolaire présente des difficultés. Comme l'ont signalé les historiens Paul-André Linteau, Jean-Claude Robert et René Durocher, «il y a lieu de croire que non seulement les statistiques relatives à cette question n'étaient pas toujours recueillies convenablement mais qu'on avait, dans certains cas, avantage à les gonfler pour des raisons financières et idéologiques ${ }^{33}$. Néanmoins, ayant pu constater le souci de précision, la manie même, qui caractérisent Viger, nous estimons que ses données sur le nombre d'écoliers sont fiables. Mais Viger parle d'écoliers «fréquentant» ou «allant» à telle(s) école(s) ou encore d'écoles «fréquentées» par tant d'enfants sans préciser s'il s'agit d'écoliers fréquentant régulièrement l'école, d'écoliers présents le jour même du recensement ou d'écoliers inscrits. La distinction est importante car l'absentéisme était élevé à l'époque et variait, entre autres, selon les saisons. Il s'agit probablement d'écoliers inscrits. Cette fréquentation «maximale» constituerait toutefois un indice significatif de l'accessibilité à l'enseignement dont nous voudrions mieux cerner ici certains aspects.

Un autre problème se pose: dans ses tableaux récapitulatifs, Viger a indiqué le nombre d'enfants âgés de 6 à 13 ans et de 6 à 17 ans, sans cependant préciser la proportion d'écoliers appartenant à ces groupes d'âge. On peut néanmoins distinguer deux taux de fréquentation scolaire: un premier taux calculé à partir de la population de 6 à 13 ans et un second portant sur la population de 6 à 17 ans. Pour les besoins de cette étude, dont le but est de mesurer et d'expliquer les taux de scolarisation des enfants considérés alors d'âge scolaire, seul le taux des 6 à 13 ans sera retenu.

Dans l'ensemble de l'île, en 1825, la fréquentation scolaire se situe, après soustraction des écoliers venant de l'extérieur de l'île et de l'étranger, à 44\% pour la catégorie des 6 à 13 ans. Déjà, elle apparaît beaucoup plus élevée que les quelque $9 \%$ trouvés en 1828 pour tout le Bas-Canada $^{34}$. Mais en milieu rural, comme le montre le tableau 3, la

33 P.-A. Linteau, R. Durocher et J.- C. Robert, Histoire du Québec contemporain, 1: De la Confédération à la crise (1867-1929) (Montréal, Boréal Express, 1979), 538.

34 L.-P. Audet, Le système scolaire..., V: 138. Les statistiques pour le Bas-Canada concernent toutefois la population âgée de 5 à 14 ans. 
fréquentation scolaire est fort différente de celle observée en milieu urbain.

TABLEAU 3

Fréquentation scolaire et ratio école/habitants selon le lieu, île de Montréal

\begin{tabular}{|l|r|r|r|r|}
\hline & \multicolumn{2}{|c|}{1825} & \multicolumn{2}{c|}{$1835^{*}$} \\
\hline Lieu & fréq. scol. & ratio é:h & fréq. scol. & ratio é:h \\
\hline & $6-13 \mathrm{a}$ & & $6-13 \mathrm{a}$ & \\
Partie rurale & $11,3 \%$ & $1: 1134$ & $22,6 \%$ & $1: 731$ \\
Partie urbaine & $71,1 \%$ & $1: 550$ & $73,3 \%$ & $1: 478$ \\
Île & $44,0 \%$ & $1: 690$ & $53,0 \%$ & $1: 543$ \\
\hline
\end{tabular}

* Les taux pour 1835 ont été calculés d'après les populations estimées.

Sources: J. Viger, Enseignement public dans le Comté de Montréal, en 1825 et Enseignement public dans le Comté de Montréal, en 1835, Fonds Verreau, ASQ.

Avec ses 335 écoliers $^{35}$ sur un potentiel de 1964 enfants de 6 à 13 ans, la partie rurale de l'île présente un taux de fréquentation scolaire de $11,3 \%$, à peine supérieur à celui du Bas-Canada à la même époque. On peut toutefois constater que la population ne dispose que de 13 écoles, soit d'une pour 1589 habitants. Qui plus est, cinq des neuf paroisses rurales sont dépourvues d'école. La rareté relative et l'éloignement des écoles, pour plusieurs enfants, peuvent expliquer en bonne partie la très faible fréquentation scolaire des jeunes ruraux.

À la suite d'une augmentation de $100 \%$ du nombre des écoliers (voir le tableau 2), la fréquentation scolaire des enfants de 6 à 13 ans a doublé en milieu rural en l'espace de dix ans ${ }^{36}$ (voir le tableau 3). Le nombre d'écoles a, lui aussi, plus que doublé, passant à 17, soit à une

35 Nous obtenons ce chiffre en soustrayant les 27 étrangers et en ajoutant les 9 écoliers «hors paroisse» des faubourgs aux 353 écoliers recensés par Viger.

36 Pour 1835, nous avons au préalable modifié certaines statistiques du tableau récapitulatif de Viger pour 1835: en premier lieu, le nombre d'écoliers de la vieille ville, et partant de la partie urbaine de l'île, a été augmenté du nombre d'élèves présents dans deux écoles découvertes tardivement par Viger. Nous avons aussi substitué au nombre d'habitants et d'enfants d'âge scolaire, obtenu par Viger d'après le taux d'accroissement annuel moyen de 1825 à 1831 (car il n'y a pas eu de recensement en 1835), les chiffres que nous avons obtenus en utilisant le taux d'accroissement annuel moyen de 1831 à 1844 . Ce taux a été retenu car il rend mieux compte selon l'historien J.-C. Robert de l'immigration britannique et de la grande épidémie de choléra qui affligea toute l'île de Montréal. Pour les faubourgs et la vieille ville, nous avons cependant dû employer celui de 1825 à 1842 car le recensement de 1831 ne distingue pas la population de la vieille ville de celle des faubourgs. Bien qu'imparfaites, ces nouvelles estimations, reprises en annexe, permettent une évaluation suffisammment réaliste de la situation. 
pour 731 habitants, un ratio qui tend à se rapprocher de celui de la partie urbaine de l'île. Seules les paroisses de la Longue-Pointe et de SainteAnne sont désormais sans maison d'enseignement. Un des trois pensionnats des religieuses et les deux écoles laïques privées recencées en 1825 n'existent plus en 1835. Par contre, une école de Fabrique, deux écoles des Sulpiciens, cinq nouvelles écoles laïques et sept écoles de syndics ont été établies depuis. C'est grâce surtout à ces dernières, qui accueillent la majorité des nouveaux inscrits, que la fréquentation scolaire connaît une telle hausse. Pour les paroisses rurales de l'île, la loi de 1829 et ses amendements semblent avoir eu un effet très bénéfique. Mais au total, la partie rurale de l'île accuse un retard considérable sur la partie urbaine.

En effet, avec 2549 écoliers ${ }^{37}$, les taux de fréquentation scolaire atteignent dans la cité plus de $71 \%$ pour les 6 à 13 ans en 1825 . C'est considérable pour l'époque. La cité dispose, il est vrai, de 41 établissements d'enseignement pour ses 22540 habitants, soit d'un pour 550 âmes. En 1835, la fréquentation scolaire augmente légèrement. On peut observer que le nombre d'écoles a augmenté davantage que la population, portant le ratio à 1:478.

La fréquentation scolaire augmente donc de façon significative dans toutes les parties de l'île de Montréal de 1825 à 1835 . Elle est et demeure cependant beaucoup plus élevée en milieu urbain qu'en milieu rural en dépit d'une hausse notable dans les paroisses rurales en particulier. Des écoles plus nombreuses entraînent, en partie du moins, cette amélioration. Ainsi, en milieu rural, l'État et, dans une moindre mesure, les Sulpiciens ont joué un rôle prédominant en ce qui concerne une plus grande disponibilité des écoles. L'augmentation significative de la fréquentation scolaire révèle également que les familles étaient plus favorables à l'instruction de leurs enfants quand celle-ci leur était facilitée. En milieu urbain, l'amélioration du réseau scolaire revient surtout à l'initiative privée laïque. Par la création de nouvelles écoles essentiellement gratuites, patronnées par des Sociétés d'éducation et de bienfaisance, la bourgeoisie montréalaise montre un souci d'instruire les défavorisés, plus nombreux depuis la décennie 1830. Sans doute, s'agit-il aussi pour elle d'accroître son prestige et son influence. On peut encore y voir l'intention d'encadrer, d'assimiler le flot d'immigrants irlandais et de contribuer ainsi à assurer l'ordre social. D'autre part, la présence de nombreuses écoles tenues par des maîtres laïcs indépendants pourrait signifier la préoccupation soutenue des parents de confier au secteur privé laïc l'éducation de leurs enfants. Ces écoles correspondaient peutêtre davantage aux attentes ou aux besoins des familles. Voyons maintenant si le souci d'instruire est le même envers les garçons et les filles.

37 Des 2703 écoliers fréquentant les institutions de la cité, nous avons retiré les 145 écoliers étrangers et les 9 venant de l'extérieur de la Paroisse de Montréal. 
On a longtemps soutenu que les filles étaient plus instruites que les garçons au 19e siècle. Elles étaient peut-être plus «savantes» mais, comme Allan Greer, nous avons trouvé qu'elles étaient moins scolarisées que les garçons dans la première moitié du siècle. Le tableau 4 montre en effet la présence, en 1825 surtout, d'une majorité significative de garçons parmi les écoliers de l'île, même en tenant compte du fait que 50,6\% des insulaires de 0 à 17 ans sont de sexe masculin ${ }^{38}$.

TABLEAU 4

Répartition des élèves selon le sexe et le lieu, île de Montréal

\begin{tabular}{|l|r|r|r|r|}
\hline & \multicolumn{2}{|c|}{1825} & \multicolumn{2}{|c|}{$1835 \%$} \\
\hline Lieu & nombre & $\%$ & nombre & $\%$ \\
\hline Partie rurale & $187 \mathrm{~g}$. & $53,0 \%$ & $367 \mathrm{~g}$. & $54,0 \%$ \\
& $166 \mathrm{f}$. & $47,0 \%$ & $313 \mathrm{f}$. & $46,0 \%$ \\
Partie urbaine & $1543 \mathrm{~g}$. & $57,1 \%$ & $2015 \mathrm{~g}$. & $51,4 \%$ \\
& $1160 \mathrm{f}$. & $42,9 \%$ & $1906 \mathrm{f}$. & $48,6 \%$ \\
Île & $1730 \mathrm{~g}$. & $56,6 \%$ & $2382 \mathrm{~g}$. & $51,8 \%$ \\
& $1326 \mathrm{f}$. & $43,4 \%$ & $2219 \mathrm{f}$. & $48,2 \%$ \\
\hline
\end{tabular}

* Dans ce tableau, nous n'avons pas soustrait les élèves étrangers et «hors paroisses» car il est difficile de savoir s'il s'agit de garçons ou de filles.

Sources: J. Viger, Enseignement public dans le Comté de Montréal, en 1825 et Enseignement public dans le Comté de Montréal, en 1835, Fonds Verreau, ASQ.

Cette inégalité apparaît tant en milieu rural qu'en milieu urbain. Il y a pourtant un nombre sensiblement égal d'écoles ouvertes aux garçons et aux filles. Il faut donc considérer l'attitude des principaux responsables de l'éducation en ce qui a trait à l'éducation des filles et des garçons, attitude perceptible à travers la répartition des écoles et des élèves selon le sexe et la catégorie (voir tableaux 5 et 6 ).

On constate, sans grande surprise, que les religieuses, soutenues ou non par les Sulpiciens, n'instruisent que des filles à l'exception de quelques garçons «trouvés» et de quelques orphelins irlandais à la petite école de l'Hôpital-Général des Soeurs Grises. Les Sulpiciens, par contre, se préoccupent davantage des garçons sans pour autant négliger les filles, car la plupart de leurs écoles et toutes celles des laïcs qu'ils «protègent» sont mixtes. Cette mixité traduit l'indépendance des Sul-

${ }^{38}$ D'après J. Viger, Tablette Statistique du Comté de Montréal, pour 1825, 2: Population du Comté d'après certaines classifications; montrant particulièrement les Enfans (sic) et distinguant les sèxes (sic), Fonds Verreau, Archives du Séminaire de Québec. 
TABLEAU 5

Répartition des écoles selon la catégorie et le sexe des élèves, île de Montréal

\begin{tabular}{|c|c|c|c|c|c|c|c|c|}
\hline \multirow[b]{2}{*}{ Catégorie } & \multicolumn{4}{|c|}{1825} & \multicolumn{4}{|c|}{1835} \\
\hline & total & éc.g & éc.f & éc. $g+f$ & total & éc.g & éc.f & éc. $g+f$ \\
\hline de l'Institution royale & 2 & 1 & & 1 & 2 & 1 & & 1 \\
\hline de Syndics & & & & & 7 & 2 & & 5 \\
\hline de Fabrique & & & & & 1 & 1 & & \\
\hline de Curé & 1 & 1 & & & 1 & 1 & & \\
\hline de l'Évêque Lartigue & 1 & 1 & & & 1 & & & 1 \\
\hline des Soeurs & 6 & & 5 & 1 & 5 & & 5 & \\
\hline des Soeurs/Sulp. & & & & & 2 & & 2 & \\
\hline des Sulpiciens & 10 & 3 & & 7 & 9 & 2 & & 7 \\
\hline $\begin{array}{l}\text { des Laïcs/Sulpiciens } \\
\text { des Sociétés }\end{array}$ & 3 & & & 3 & 5 & 1 & & 6 \\
\hline des Habitants & 1 & & & 1 & & & & \\
\hline des Églises & 1 & 1 & & & 2 & 1 & & \\
\hline des Laïcs privés & $28 *$ & 2 & 3 & 23 & 47 & 5 & 7 & 35 \\
\hline Total & $53 *$ & 9 & 8 & 36 & 89 & 14 & 14 & 61 \\
\hline
\end{tabular}

* Il y a une école dont on ignore le sexe des élèves.

Sources: J. Viger, Enseignement public dans le Comté de Montréal, en 1825 et Enseignement public dans le Comté de Montréal, en 1835, Fonds Verreau, ASQ.

TABLEAU 6

Répartition des élèves selon le sexe et la catégorie de l'école, île de Montréal

\begin{tabular}{|l|r|r|r|r|}
\hline & \multicolumn{2}{|c|}{1825} & \multicolumn{2}{|c|}{1835} \\
\hline Catégorie & garçons & filles & garçons & filles \\
\hline de l'Institution royale & 78 & 8 & 49 & 6 \\
de Syndics & & & 144 & 75 \\
de Fabrique & 60 & & 19 & \\
de l'Évêque Lartigue & 28 & & 143 & 69 \\
de Curé & 7 & 434 & 21 & \\
des Soeurs & 595 & 239 & & 353 \\
des Soeurs/Sulp. & & & 786 & 272 \\
des Sulpiciens & 427 & 215 & 665 & 256 \\
des Laïcs/Sulpiciens & 16 & 8 & 107 & 61 \\
des Sociétés & 70 & & 669 \\
des Habitants & 446 & 415 & 669 & 738 \\
des Églises & & & 23 \\
des Laïcs privés & & & & \\
\hline
\end{tabular}

Sources: J. Viger, Enseignement public dans le Comté de Montréal, en 1825 et Enseignement public dans le Comté de Montréal, en 1835, Fonds Verreau, ASQ. 
piciens à l'égard de Mgr Lartigue qui enjoignait à ses curés de refuser les sacrements aux enfants, parents et maîtres qui l'encourageaient ${ }^{39}$. En fait, les Sulpiciens séparent plutôt leurs élèves selon la langue. En 1825, l'Évêque n'admet que des garçons dans son école de SaintJacques. En 1835, il y accepte des filles mais, fidèle à ses principes, il les regroupe dans une classe séparée de celles des garçons.

L'État, présent dans les écoles de l'Institution royale et, surtout, dans les écoles de syndics, se soucie, semble-t-il, davantage des garçons. Il ne paraît, par ailleurs, guère influencé par les principes de Mgr Lartigue, puisque les filles sont instruites aussi bien dans des classes mixtes que séparées. Mais la mixité révèle également la situation précaire des paroisses rurales trop pauvres pour engager un deuxième mâ̂tre ou une deuxième maîtresse. Dans les écoles de syndics, l'on ne retrouve des classes séparées pour les garçons et les filles que lorsque le nombre d'élèves dépasse la quarantaine.

Les écoles des Sociétés d'éducation et de bienfaisance sont ouvertes tant aux filles qu'aux garçons. Le nombre de leurs élèves de sexe masculin l'emporte toutefois largement sur celui de sexe féminin. Nos sources ne nous permettent toutefois pas de savoir si cette inégalité résulte de la politique des administrateurs ou de l'attitude des familles. Enfin, les écoles laïques privées, plus fragiles et assujetties aux voeux des parents, se veulent accueillantes pour l'un ou l'autre sexe. Les trois quarts sont mixtes. Mais dans les «grandes écoles» ${ }^{40}$ de cette catégorie, la mixité n'est pratiquée, en 1825 comme en 1835 , qu'une fois sur deux. Et, si c'est le cas, les filles constituent la très grande majorité des élèves. La seule «grande école» de la partie rurale de l'île, l'école de l'Institution royale de la paroisse de Lachine, compte bien huit filles parmi les quarante élèves qu'elle déclare en 1825. En règle générale, toutefois, quand les parents veulent et peuvent offrir à leurs filles une instruction plus poussée, ils préfèrent des établissements qui leur sont avant tout destinés. L'essentiel qui se dégage cependant de ces statistiques, c'est que la fréquentation scolaire des garçons est supérieure à celle des filles au milieu des décennies 1820 et 1830 . Et ce phénomène semble relié, dans une certaine mesure, à l'attitude et à la philosophie éducative des grands responsables de l'enseignement.

La dimension ethnique dans l'enseignement revêt, nous l'avons vu, un intérêt considérable au Bas-Canada dans le premier tiers du $19 \mathrm{e}$ siècle, et plus particulièrement à Montréal où la composition ethnique et, partant, l'équilibre linguistique sont modifiés au tournant des années 1830 par l'arrivée massive d'immigrants britanniques.

39 Y. Majerus, L'éducation dans le diocèse de Montréal d'après la correspondance des deux premiers évêques, Mgr J.-J. Lartigue et Mgr I. Bourget de 1820 à 1867, Thèse de doctorat (histoire), Université McGill, 1971, 62-63.

40 Il s'agit d'écoles de niveau «secondaire». 
TABLEAU 7

Répartition des élèves selon la langue d'enseignement et le lieu, Île de Montréal

\begin{tabular}{|l|r|r|r|r|r|r|}
\hline & \multicolumn{3}{|c|}{1825} & \multicolumn{3}{c|}{1835} \\
\hline Lieu & $\begin{array}{r}\text { écoles } \\
\text { franç. }\end{array}$ & $\begin{array}{r}\text { écoles } \\
\text { ang. }\end{array}$ & $\begin{array}{r}\text { écoles } \\
\text { fr. et ang. }\end{array}$ & $\begin{array}{r}\text { écoles } \\
\text { franc. }\end{array}$ & $\begin{array}{r}\text { écoles } \\
\text { angl. }\end{array}$ & $\begin{array}{r}\text { écoles } \\
\text { fr. et ang. }\end{array}$ \\
\hline Paroisses rurales & 5 & 1 & 1 & 12 & 3 & 2 \\
Divisions & 2 & 3 & 1 & 1 & 2 & 3 \\
Faubourgs & 5 & 15 & 4 & 5 & 32 & 9 \\
Vieille ville & 3 & 9 & 5 & 3 & 13 & 4 \\
Île & 15 & 28 & 11 & 21 & 50 & 18 \\
\hline
\end{tabular}

Sources: J. Viger, Enseignement public dans le Comté de Montréal, en 1825 et Enseignement public dans le Comté de Montréal, en 1835, Fonds Verreau, ASQ.

D'après nos sources, on ne peut estimer de façon précise la fréquentation scolaire des Canadiens français et des anglophones de l'île, l'origine ethnique des élèves n'ayant pas toujours pu, selon Viger, être obtenue des maîtres ${ }^{41}$. À l'aide des tableaux 7 et 8 , il est toutefois possible d'apprécier la possibilité offerte aux enfants des deux grands groupes linguistiques de s'instruire dans leur langue. Précisons que le tableau 8 nous donne l'origine ethnique et le lieu de naissance des habitants de la cité, mais l'on peut en déduire qu'il n'existe pratiquement que deux grands ensembles linguistiques à l'époque qui nous concerne: le groupe francophone, essentiellement représenté par les Canadiens français, et le groupe des anglophones nés au Bas-Canada ou dans les colonies britanniques d'Amérique du Nord (Terre-Neuve, NouvelleÉcosse, Nouveau-Brunswick, Haut-Canada) ou encore aux États-Unis, puisqu'il y a tout lieu de croire que ces deux dernières catégories sont anglophones.

Au premier regard, ce qui étonne dans le tableau 7, c'est le grand nombre, plus de la moitié, d'écoles de l'île où l'enseignement se donne uniquement en anglais. Ces écoles sont deux fois plus nombreuses que les écoles exclusivement françaises. Il est également intéressant de constater la proportion relativement élevée, $20 \%$ en 1825 et 1835 , d'écoles bilingues. En milieu rural, la majorité canadienne-française dispose d'une majorité d'établissements de sa langue ou bilingues. Dans

${ }^{41}$ J. Viger, Enseignement public..., en 1835, 46. 
TABLEAU 8

Répartition de la population montréalaise selon l'origine ethnique et le lieu de naissance

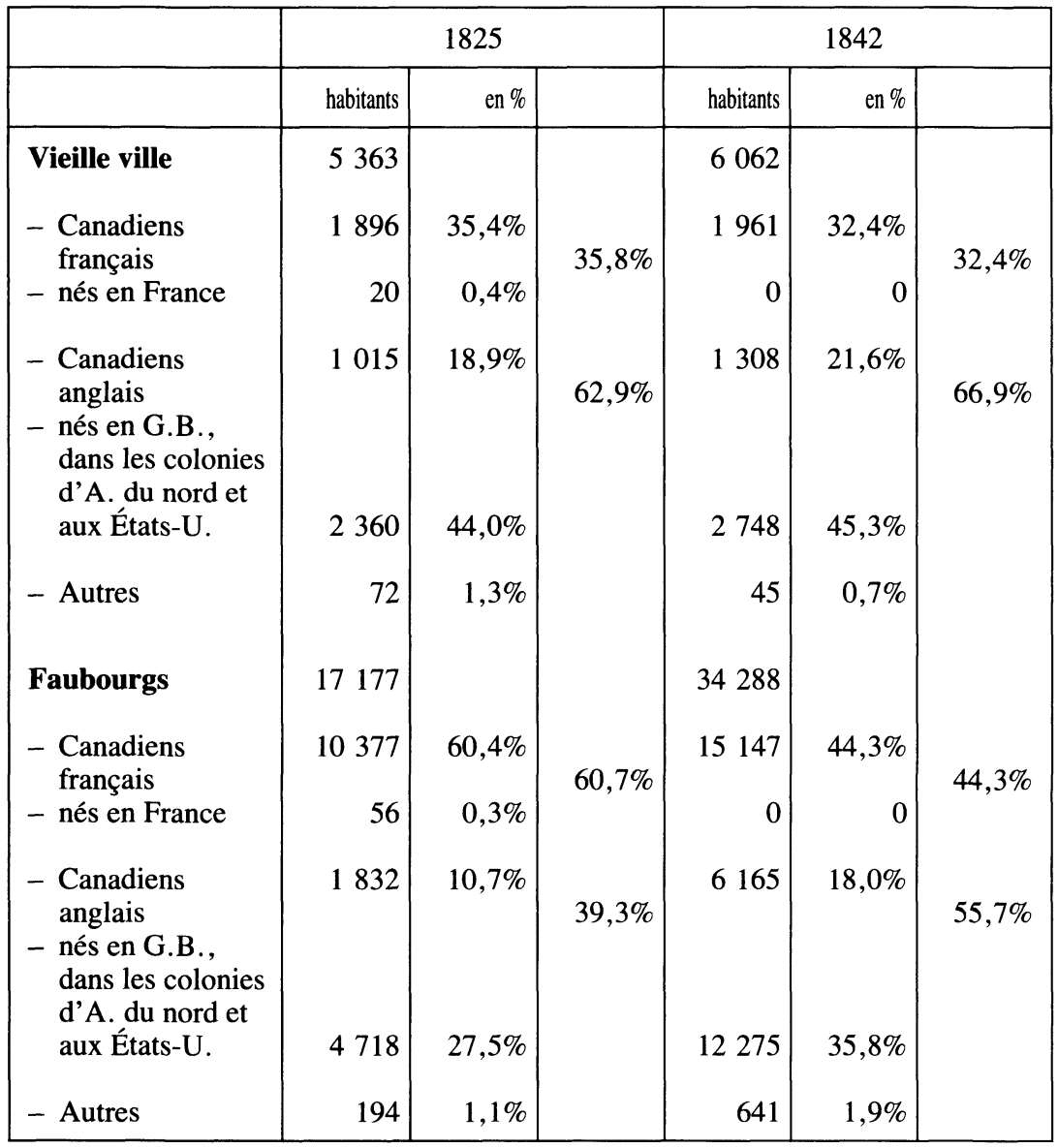

Sources: J.-C. Robert, Montréal 1821-1871. Aspects de l'urbanisation, thèse de doctorat (histoire), École des Hautes Études en sciences sociales (Université de Paris I), 1977, 391-392.

la «vieille ville», où ils forment $35,8 \%$ en 1825 et sans doute quelque 33 ou $34 \%$ en 1835 des habitants, les francophones semblent relativement bien nantis en ce domaine. Dans l'ensemble des faubourgs cependant, on note, particulièrement pour 1835 , une sur-représentation des écoles anglaises. Pour comprendre cette inégalité, examinons l'attitude des principaux responsables de l'enseignement face à la dualité linguistique et aux modifications de l'équilibre ethnique de la cité. 
De tradition britannique et régies par des Conseils d'administration majoritairement anglophones ${ }^{42}$, les Sociétés d'éducation et de bienfaisance offrent toutes, à l'exception de la toute nouvelle école des Dames de la Charité, un enseignement en anglais. En 1835, elles instruisent en cette langue plus du quart des écoliers que compte la cité. Elles sont donc dans une bonne mesure à l'origine de la disparité entre les deux groupes linguistiques en ce qui a trait à la disponibilité des écoles.

Toutefois, les écoles laïques privées sont aussi largement responsables de cette disparité qui s'accroît. En 1825, neuf établissements de cette catégorie se déclarent déjà unilingues anglais et un seul ne dispense ses cours qu'en français. Dix ans plus tard, 34 des 40 écoles indépendantes instruisent, en ce qui est maintenant la langue de la majorité des habitants de la cité, 1112 enfants de la vieille ville et surtout des faubourgs.

Mais l'Église n'est pas en reste. Ainsi, en 1825, l'école de l'évêque Lartigue est uniquement française. Son faubourg est peuplé à plus de $65 \%$ de Canadiens français. Fermement convaincu de la nécessité d'apprendre l'anglais - «en autres choses que tout le monde s'applique à l'anglais», écrivait-il au directeur du Séminaire de Saint-Hyacinthe ${ }^{43}$ - il ajoute à son école, en 1835 , deux classes anglaises et une classe bilingue fréquentées cependant par des enfants presqu'exclusivement canadiens-français. Bien que davantage concernées par l'éducation des filles francophones, les religieuses de la Congrégation n'en ont pas moins, dès 1825, une classe anglaise sur les deux que compte leur pensionnat de la vieille ville. Les Sulpiciens, eux, se révèlent soucieux, dès 1825 , de l'instruction de leurs jeunes paroissiens anglophones, car une de leurs huit écoles de la cité est unilingue anglophone et trois sont bilingues. En 1835, les laïcs dont ils assurent la «protection» se chargent davantage des anglophones avec une école anglaise et deux bilingues, notamment au faubourg Sainte-Anne où s'installent bon nombre d'immigrants irlandais.

La population anglophone de l'île, de la cité en particulier, pouvait donc bénéficier, dans les années 1820 , d'un nombre d'écoles supérieur à celui dont disposait la communauté francophone. Cet avantage, qui croît dans les années 1830, explique pour une bonne part le taux de scolarisation plus élevé des anglophones dont faisait état Allan Greer. $\mathrm{Au}$-delà des luttes de pouvoir pour le contrôle de l'instruction primaire, le souci de certains membres de la bourgeoisie anglophone de l'île d'inculquer leurs valeurs par l'intermédiaire de l'école nous semble bien à l'origine de cette disparité.

\footnotetext{
42 The Montreal Almanach or Lower Canada Register de 1829, 1830 et 1831 (Montreal, Robert Armour, 1828, 1829 and 1831), 1: 134-139, 142-143; 2: 152-157; 3: 142-149.

43 Cité par L.-P. Audet, Histoire de l'enseignement..., $1: 375$.
} 


\section{FRÉQUENTATION SCOLAIRE ET COUT DE L'INSTRUCTION}

Le coût mensuel moyen des cours s'élève à $1,13 \$$ en 1825 et à $1,22 \$$ en $1835^{44}$. À l'échelle de l'île, l'écart entre les deux recensements est minime et peu significatif. Mais ce faible écart cache des variations importantes. Les frais exigés pour les études varient énormément d'une école à l'autre en fonction notamment du nombre et de la complexité des matières enseignées. Les qualifications et la réputation des maîtres contribuent aussi, sûrement, à faire fluctuer les frais. La localisation des écoles intervient également.

TABLEAU 9

Coût mensuel moyen, en dollars, des études selon le lieu, île de Montréal

\begin{tabular}{|l|c|c|}
\hline Lieu & 1825 & 1835 \\
\hline Paroisses rurales & 0,50 & 0,38 \\
Divisions & 0,67 & 0,43 \\
Faubourgs & 0,73 & 1,38 \\
Vieille ville & 2,19 & 1,86 \\
île & 1,13 & 1,22 \\
\hline
\end{tabular}

Sources: J. Viger, Enseignement public dans le Comté de Montréal, en 1825 et Enseignement public dans le Comté de Montréal, en 1835, Fonds Verreau, ASQ.

D'après le tableau 9 , les parents doivent en effet débourser davantage en milieu urbain et de plus en plus à mesure que l'on se rapproche du coeur de la cité. Les faubourgs et la vieille ville ont évidemment davantage de «grandes écoles». Il reste qu'il en coûte plus cher de fréquenter les écoles de la ville que celles de la campagne. Le même écart existe pour les frais de pension. Dans les paroisses rurales, le coût annuel de la pension, exigée en espèces et en nature (minots de blé et cordes de bois de chauffage), et des études s'élève en moyenne à $26,10 \$$ en 1825 et à $32,31 \$$ en 1835 . Dans la cité, ces frais se montent, en excluant les orphelinats, à $124,33 \$$ puis à $153,13 \$$. Certains pensionnats privés demandent jusqu'à $160 \$$ et même $200 \$$ par année.

Le tableau 10 révèle des écarts considérables dans les frais demandés par les différents établissements. Ainsi, toutes les écoles de Sociétés sont gratuites, du moins pour la très grande majorité des

44 Pour 1825, ce coût moyen a été établi d'après la somme des coûts des études de 51 des 54 écoles; pour 1835, il a été calculé d'après les coûts de 88 des 89 écoles. Nous avons par ailleurs considéré ce qu'il en coûte pour l'enseignement terminal de l'établissement. D'autre part, les prix sont indiqués en livres canadiennes ou courantes de Halifax dans les documents. Nous les avons convertis, comme d'ailleurs les coûts exigés en nature, en dollars de l'époque. 
TABLEAU 10

Coût mensuel moyen des études selon la catégorie des écoles, île de Montréal

\begin{tabular}{|l|r|r|}
\hline Catégorie & 1825 & 1835 \\
\hline de l'Institution royale & $\$ 3,08$ & $\$ 2,50$ \\
de Syndics & & $\$ 0,35$ \\
de Fabrique & 0 & $\$ 0,34$ \\
du Curé & 0 & $\$ 0,40$ \\
de l'Évêque Lartigue & $\$ 0,15$ & 0 \\
des Soeurs* & $\$ 0,13$ \\
des Soeurs/Sulpiciens & $\$ 0,42$ & 0 \\
des Sulpiciens & 0 & $\$ 0,39$ \\
des Laïcs/Sulpiciens & 0 & $\$ 1,05$ \\
des Sociétés & $\$ 4,00$ & 0 \\
des Habitants & $\$ 1,55$ & $\$ 2,00 * *$ \\
des Églises & & $\$ 1,84$ \\
des Laïcs privés & \\
\hline
\end{tabular}

* À l'exclusion de leur Pensionnat de la vieille ville où le coût de l'enseignement seul n'est pas indiqué.

** L'école de la vieille ville coûte $\$ 4,00$ et celle des faubourgs est gratuite.

Sources: J. Viger, Enseignement public dans le Comté de Montréal, en 1825 et Enseignement public dans le Comté de Montréal, en 1835, Fonds Verreau, ASQ.

élèves ${ }^{45}$. Cette gratuité explique en bonne partie que la National School, la Recollet School et la British and Canadian School soient les écoles montréalaises les plus populeuses. Il en coûte aussi moins cher de fréquenter les écoles privées soutenues par l'Église catholique que celles tenues par des laïcs. En ce qui concerne les écoles de l'État, les prix des écoles de l'Institution royale, toutes deux de niveau «secondaire» en 1825 , ne sont nullement comparables à ceux des écoles de syndics. La baisse du coût mensuel moyen des études qui apparaît en 1835 dans les paroisses rurales et les divisions est due à l'ouverture d'écoles de syndics pour les premières et des Sulpiciens pour les secondes.

L'enseignement est parfois gratuit chez les Sulpiciens, mais ceuxci exigent la contribution des parents dans la plupart de leurs maisons. Les laïcs qu'ils «protègent» exigent, pour leur part, des frais qui se rapprochent de ceux exigés dans les écoles laïques privées. Serait-ce pour les Sulpiciens un moyen discret, déguisé, de réserver une partie de leurs établissements aux seuls bien nantis? On peut aussi penser que leur «protection» est symbolique et sert surtout aux maîtres à assurer leur réputation auprès de leur clientèle des faubourgs. Chez les religieuses et les presbytériens, il semble bien exister un double «stan-

45 Selon Viger, Enseignement public... 1825, 15, des frais de $0,15 \$$ à $0,67 \$$ par mois seraient demandés aux parents qui peuvent payer. La plupart ne paieraient cependant rien. 
dard». Ainsi, les frais de pension et d'enseignement s'élèvent à $50 \$$ puis à $100 \$$ par an dans le pensionnat de premier niveau de la vieille ville, mais les religieuses n'exigent rien de la très grande majorité de leurs élèves dans les «Petites écoles françaises», dans le grand externat voisin du pensionnat. De même, l'Église presbytérienne tient-elle aussi une «grande école» à $4 \$$ par mois dans la vieille ville et un établissement gratuit de niveau élémentaire dans les faubourgs.

Constituant sans doute la principale ou l'unique source de revenus de leurs dirigeants, les frais exigés par les laïcs indépendants paraissent en moyenne relativement élevés. Mais plusieurs laïcs affichent des frais relativement modestes: $0,50 \$$ et même moins par mois pour un curriculum restreint, il faut le dire. Il pouvait s'agir de maîtres improvisés en quête d'un revenu d'appoint ou temporaire leur permettant de s'établir à Montréal ou de partir vers le Haut-Canada ou les États-Unis. Par ailleurs, l'importante différence de coût constatée en 1825 entre la vieille ville et les faubourgs se rétrécit considérablement en 1835: plusieurs écoles laïques de niveau «secondaire» sont créées dans les faubourgs en même temps que deux écoles gratuites de Sociétés dans la vieille ville. Celle-ci connaît en outre une baisse sensible des frais de ses écoles laïques, une baisse due, pensons-nous, à la concurrence qui rend plus difficile le recrutement des élèves dans cette zone urbaine de quelque 5700 habitants.

Nous avons tenté de voir, au moyen du tableau 11, ce que représentent ces coûts pour les familles. Pour maintes familles des paroisses rurales, le coût de l'instruction nous apparaît élevé. En 1825, bon nombre

TABLEAU 11

Quelques exemples de salaires, revenus et prix ayant cours au Bas-Canada dans les décennies 1820 et 1830

\begin{tabular}{|lr|lr|}
\hline \multicolumn{2}{|c|}{ Salaires et revenus } & \multicolumn{2}{c|}{ Prix de certaines denrées } \\
\hline Cultivateur & $\$ 316,00 / \mathrm{an} *$ & minot de blé** & $\$ 1,00$ \\
Employé agricole & $\$ 8,00 / \mathrm{ms}$ & corde de bois mél.** & $\$ 2,50$ \\
Maçon, charp. forger. & $\$ 1,00$ à $\$ 2,00 / \mathrm{jr}$ & minot de p. de terre & $\$ 0,20$ \\
Journalier & $\$ 0,50$ à $\$ 0,80 / \mathrm{jr}$ & livre de boeuf** & $\$ 0,05$ \\
Domestiques (hom.) & $\$ 5,20$ à $7,20 / \mathrm{ms}$ & poêle à chauffage & $\$ 12$ à $\$ 40$ \\
Conseiller législatif & $\$ 2000$ à $\$ 3000 /$ an & Piano & $\$ 200,00$ \\
\hline
\end{tabular}

* Ce revenu constitue la médiane des revenus des chefs de famille de cultivateurs de Laprairie. On peut supposer que le revenu moyen de ces cultivateurs se rapproche de cette médiane.

** Prix du marché à Montréal.

Sources: Recensement de 1831; La Minerve, juin à septembre 1829; G. Bervin, «Environnement matériel et activités économiques des conseillers exécutifs et législatifs à Québec, 1810-1830», Bulletin d' histoire de la culture matérielle, 17 (Ottawa, Musées nationaux du Canada, 1983): 4562; L. Blanchette-Lessard et N. Daigneault-Saint-Denis, Groupes sociaux, patriotes et les rébellions de 1837-1838: idéologie et participation, Thèse de maîtrise (histoire), Université du Québec, 1975, 158-159. 
de ces familles étaient sans doute incapables d'offrir à leurs enfants les «grandes écoles» et pensionnats de la cité et même certaines écoles primaires locales. Aussi, les écoles de syndics ont contribué, autant par leur moindre coût que par leur présence, à l'augmentation de la fréquentation scolaire en milieu rural en 1835 . La population des campagnes n'était sans doute pas aussi indifférente à l'égard de l'instruction que le prétendaient certains contemporains. Des revenus limités devaient bien souvent l'en détourner.

Dans la cité, les frais exigés, souvent très élevés, n'empêchent sans doute pas la grande bourgeoisie d'affaires, majoritairement anglophone par ailleurs ${ }^{46}$, d'offrir à ses fils et filles une instruction de haut niveau. Pour les enfants des travailleurs qualifiés, l'instruction paraît accessible, une instruction de moindre niveau sans doute. Mais, pour la catégorie des journaliers et des domestiques, relativement peu rémunérée, et pour les premiers, particulièrement affectés par le chômage saisonnier qui afflige l'économie montréalaise au cours de la période que nous étudions ${ }^{47}$, les frais exigés dans de nombreuses écoles s'avèrent, selon nous, un obstacle à la fréquentation scolaire.

La gratuité des études constitue en revanche une incitation efficace à la fréquentation scolaire. En effet, la proportion élevée d'écoliers instruits gratuitement dans l'île, $41,8 \%$ en 1825 et $43,3 \%$ en 1835 , a probablement contribué aux taux relativement élevés de scolarisation qu'on y retrouve. Mais ici encore les jeunes ruraux sont désavantagés

TABLEAU 12

Nombre et pourcentage des écoliers instruits gratuitement selon le lieu, Île de Montréal

\begin{tabular}{|l|r|r|r|r|}
\hline & \multicolumn{2}{|c|}{1825} & \multicolumn{2}{c|}{1835} \\
& écoliers instruits gratuitement & écoliers instruits gratuitement \\
\hline & nombre & $\%$ & nombre & $\%$ \\
\hline Paroisses rurales & 37 & $17,3 \%$ & 57 & $13,2 \%$ \\
Divisions & 37 & $26,6 \%$ & 14 & $5,6 \%$ \\
Partie rurale & 74 & $21,0 \%$ & 71 & $10,4 \%$ \\
Faubourgs & 479 & $33,9 \%$ & 939 & $40,8 \%$ \\
Vieille ville & 723 & $56,1 \%$ & 982 & $60,6 \%$ \\
Partie urbaine & 1202 & $44,5 \%$ & 1921 & $49,0 \%$ \\
Île & 1276 & $41,8 \%$ & 1992 & $43,3 \%$ \\
\hline
\end{tabular}

Sources: J. Viger, Enseignement public dans le Comté de Montréal, en 1825 et Enseignement public dans le Comté de Montréal, en 1835, Fonds Verreau, ASQ.

\footnotetext{
46 F. Ouellet, «Structure des occupations et ethnicité dans les villes de Québec et Montréal (1819-1844)», Eléments d' histoire sociale..., 182-202.

47 J.-P. Bernard, P.-A. Linteau et J.-C. Robert, «La structure...», 397.
} 
comme on peut le voir au tableau 12. En milieu rural, la gratuité scolaire dont se chargent avec de plus en plus de parcimonie les écoles de syndics ne peut être évoquée pour justifier les progrès qui y apparaissent au milieu des années 1830 au chapitre de la fréquentation scolaire.

En milieu urbain, près d'un écolier sur deux et dix fois plus d'enfants d'âge scolaire bénéficient de la gratuité scolaire. Cette gratuité, assurée par l'évêque Lartigue, les religieuses, les Sulpiciens et, surtout, par les Sociétés d'éducation et de bienfaisance, a sans doute été un des grands facteurs de la forte fréquentation des écoles et de la hausse que celle-ci connaît en 1835 .

On peut également mettre au crédit de la gratuité scolaire la réduction, en 1835, de l'important écart qui existe en 1825 entre la scolarisation des filles et celle des garçons (voir tableau 4). En effet, le rattrapage qu'effectuent les jeunes citadines résulte, dans une bonne mesure, de l'ouverture en 1833 de deux nouvelles écoles gratuites par les Sulpiciens et les religieuses de la Congrégation de Notre-Dame dans les faubourgs populeux de Saint-Laurent et de Sainte-Marie. Avec ces 272 nouvelles admissions, $58 \%$ des élèves instruits gratuitement au milieu des années 1830 étaient des filles alors que dix ans plus tôt celles-ci constituaient seulement le tiers des élèves non-payants.

Par ailleurs, un jeune citadin a plus de chance d'être instruit gratuitement s'il étudie en anglais, comme le montre le tableau 13. Près de $60 \%$ des élèves qui ne paient pas fréquentent en effet des écoles anglaises en 1825. Les Sociétés d'éducation et de bienfaisance, par souci peut-être de socialiser les jeunes immigrants, en accueillent la

TABLEAU 13

Répartition des écoliers instruits gratuitement selon la langue d'enseignement, Cité de Montréal

\begin{tabular}{|l|c|c|}
\hline & 1825 & 1835 \\
\hline en français & 470 & 674 \\
en anglais & 712 & 1100 \\
dans les 2 langues & 0 & 147 \\
\hline
\end{tabular}

Sources: J. Viger, Enseignement public dans le Comté de Montréal, en 1825 et Enseignement public dans le Comté de Montréal, en 1835, Fonds Verreau, ASQ.

grande majorité. Dans les écoles françaises, Mgr Lartigue ainsi que les Sulpiciens et les religieuses (lorsqu'ils sont associés) se partagent cette charge. Chez les francophones, la gratuité scolaire c'est donc surtout l'affaire de l'Église. Celle-ci retient ou attire également par ce moyen une partie des immigrants anglophones et même des Canadiens français 
dans ses classes bilingues et anglaises. Au total, cependant, le groupe anglophone apparaît davantage favorisé au niveau de la gratuité scolaire. Celle-ci peut donc aussi être considérée comme un facteur explicatif de la plus grande scolarisation de la communauté anglophone bascanadienne constatée par Allan Greer.

\section{CONCLUSION}

Cet article voulait surtout montrer, à travers l'existence d'un lien entre la fréquentation scolaire et le nombre d'écoles dont disposaient l'ensemble de la population ainsi que les diverses catégories de clientèles étudiantes, l'importance de la disponibilité des écoles en matière de fréquentation scolaire au cours de la période 1801-1836. Il voulait également souligner l'importance du coût des études et de la gratuité scolaire dans la fréquentation des écoles et, partant, dans l'accessibilité à l'instruction. Tout en reconnaissant l'influence des mentalités, nous constatons la prédominance de l'offre institutionnelle et des facteurs économiques dans la fréquentation scolaire au Bas-Canada.

Dans un contexte de vive rivalité, ce sont les principaux éducateurs du temps qui ont surtout défini l'accessibilité à l'école. Par leur poids politique et économique et selon leur philosophie éducative, ils ont en effet souvent déterminé la densité du réseau scolaire et le coût de l'enseignement. En définitive, l'accès à l'école fut dans l'ensemble davantage facilité aux citadins qu'aux ruraux, aux garçons qu'aux filles, aux anglophones qu'aux francophones et, en milieu rural surtout, aux bien nantis qu'aux mal nantis. Ces inégalités vis-à-vis de l'accessibilité à l'instruction pourraient bien être à l'origine des mêmes disparités retrouvées au Québec au milieu du 19e siècle au niveau de l'alphabétisation.

Grâce à des sources nouvelles, notre étude a également mis en lumière un réseau d'écoles relativement dense et surtout diversifié dans l'île de Montréal dans les décennies 1820 et 1830 . Cette diversité remet en question notre perception d'un système d'enseignement essentiellement aux mains de l'Église, de l'État et des Sociétés d'éducation à la fin de la période 1801-1836. Le cas des nombreuses écoles laïques retrouvées mérite de plus amples recherches. La présence de telles écoles traduit-elle surtout la méfiance des parents à l'égard des établissements d'enseignement public ou correspond-elle plutôt à une stratégie de survie des immigrants? Au Bas-Canada, s'agit-il d'un phénomène uniquement montréalais, essentiellement urbain et relié à la communauté anglophone? Quelle importance auront ces écoles dans l'évolution du système scolaire québécois qui s'implante pour de bon dans le CanadaEst à partir des années 1840 ? La réponse à ces questions devrait ajouter à notre connaissance du système scolaire bas-canadien et québécois ainsi que de la société montréalaise du $19 \mathrm{e}$ siècle. 


\section{ANNEXE}

Corrections apportées au tableau concernant l'«Enseignement public» en 1835*

\begin{tabular}{|c|c|c|c|c|c|c|c|c|c|c|c|}
\hline \multirow[t]{2}{*}{ Lieu } & Habitants & \multicolumn{2}{|c|}{ Enfants } & \multicolumn{4}{|c|}{ Écoliers } & \multicolumn{2}{|c|}{ Résidant } & \multicolumn{2}{|c|}{ «Petites écoles» } \\
\hline & & 6 à 13 ans & 6 à 17 ans & Garçons & Filles & Pens. & Ext. & ds Par. H & et Et. & franç. & angl. \\
\hline $\begin{array}{l}\text { Paroisses } \\
\text { rurales }\end{array}$ & 12759 & 2679 & 3844 & 240 & 191 & & & & & & \\
\hline Divisions & 4071 & 708 & 1053 & 127 & 122 & & & & & & \\
\hline Faubourgs & 25771 & 4306 & 6286 & 1090 & 1211 & & & & & & \\
\hline Vieille ville & 5762 & 770 & 1379 & 925 & 695 & 139 & 1419 & 1602 & 18 & 3 & 6 \\
\hline Île & 48363 & 8463 & 12562 & 2382 & 2219 & 434 & 4414 & 4361 & 117 & 18 & 32 \\
\hline
\end{tabular}

* Le nombre total d'habitants et d'enfants a été estimé d'après le taux d'accroissement annuel moyen de 1831 à 1844 pour les paroisses rurales et les «divisions» et de 1825 à 1842 pour la vieille ville et les faubourgs. Les chiffres concernant les écoliers et les «petites écoles» tiennent compte de l'ajout des données des deux écoles découvertes tardivement par Jacques Viger.

Sources: J. Viger, Enseignement public dans le Comté de Montréal, en 1825 et Enseignement public dans le Comté de Montréal, en 1835 , Fonds Verreau, ASQ; Recensements du Bas-Canada de 1831, 1842 et 1844. 